\title{
Le réseau de distribution d'eau potable à Paris : mesures de débit in situ, comparaison de débitmètres installés à demeure à des étalons
}

\author{
O. Périllat et J. Hérigault \\ E.D.F.-D.T.G. Grenoble
}

\section{Le contexte}

La Ville de Paris est alimentée en eau potable par l'intermédiaire de trois Sociétés. La première, la S.A.G.E.P., assure l'acheminement de l'eau depuis les captages jusqu'à des bassins de stockage ou directement au réseau en périphérie de Paris.

Les deux autres Compagnies (la C.E.P. et la S.P.E.) se sont vu confier la gestion de la distribution dans la ville. Elles se partagent Paris, le fleuve Seine servant de frontière naturelle.

Le réseau n'est pas symétrique et l'eau arrive en grande partie par la Rive Gauche. Il y a donc nécessité de transferts vers la Rive Droite. Ces échanges inévitables se font par l'intermédiaire de conduites de différents diamètres (de $\varnothing 400$ à $\varnothing 1500 \mathrm{~mm}$ ) logées sous plusieurs ponts de la Capitale.

Ces transferts entre les deux Sociétés sont naturellement comptabilisés puisqu'ils ouvrent droit à facturation.

Le volume transité est comptabilisé à partir du débit, lui-même mesuré par méthode ultrasonore. Plus d'une centaine de débitmètres ultrasonores majoritairement monocordes sont ainsi installés pour le contrôle des volumes transités mais également pour l'exploitation du réseau. Ils sont de marque Ultraflux.

A l'usage, des doutes sur la qualité des mesures de débit sont apparus ; les deux parties ont alors demandé conjointement une expertise. E.D.F.-D.T.G. a été choisi pour réaliser des mesures de référence.

Nous avons tout d'abord réalisé un contrôle dimensionnel précis des différentes grandeurs géométriques sur de nombreuses sections d'installation des débitmètres ultra- sonores. En effet, ce type de débitmètre est très sensible aux paramètres géométriques d'installation (diamètre, distance entre sondes, ...). Ces contrôles ont été effectués sur une trentaine de sections de mesure au total.

Une grande partie de l'expertise s'est ensuite basée sur des mesures comparatives entre les systèmes en place et des étalons mis en œuvre sur site.

Dans ce cadre, E.D.F.-D.T.G. a appliqué diverses méthodes de mesure comme le jaugeage aux moulinets sur quatre sections de mesure ultrasonore. Un autre essai a permis d'étalonner un débitmètre à partir de la mesure du débit refoulé par une pompe en appliquant la méthode thermodynamique à la machine.

Ces méthodes, définies par des normes internationales, serviront de référence absolue.

\section{La débitmétrie ultrasonore}

Le principe des débitmètres à ultrasons est basé sur la composition vectorielle de la célérité du son avec la vitesse de l'eau. Le débitmètre mesure les temps de parcours aller et retour des ondes ultrasonores d'une sonde à l'autre, la différence des deux temps de transit caractérisant, on va le voir, le débit.

Les débitmètres investigués sont de deux types: Ultraflux UF 320 et UF 321 . Les sondes utilisées sont identiques, seul le traitement de l'information est différent (fig. 1).

Le débit calculé peut être écrit sous la forme :

$$
Q_{U S}=k \cdot\left(\Delta t / t^{2}\right) \cdot L^{2} \cdot D^{2} / d .
$$

Drinkable water supply network of Paris :

in situ flow measurement, comparison between fixed flowmeters and master instruments

Ultrasonic flowmeters setting up for water networks management is difficult. It is necessary to comply with the manufacturer's start up processes. Measure differences were observed when comparing measurement on drinkable water supply network of Paris with E.D.F.-D.T.G. master instruments references. The choice of measure and probes setting up in the pipe is at the origin of the differences. 


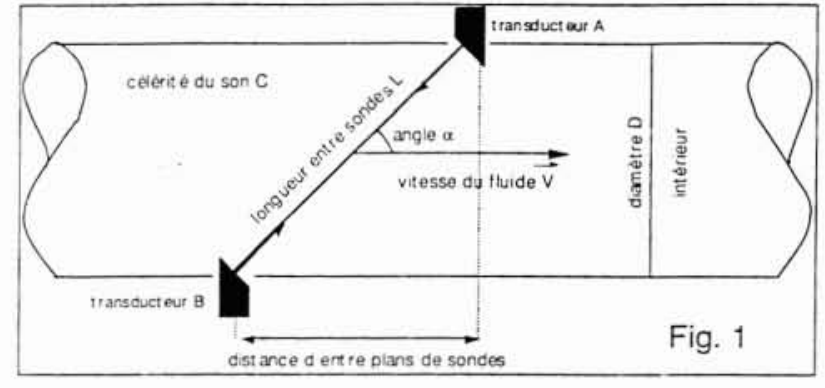

1. Principe de la débitmétrie ultrasonore.

Le débit $Q_{U S}$ dépend donc :

- des mesures intrinsèques de temps de transit $\mathrm{t}_{\mathrm{AB}}$ et $\mathrm{t}_{\mathrm{BA}}$ et les valeurs qui en découlent :

$$
\begin{array}{ll}
\Delta \mathrm{t}=\mathrm{t}_{\mathrm{AB}}-\mathrm{t}_{\mathrm{BA}} & \text { différence de temps de transit } \\
t=\left(t_{A B}+t_{B A}\right) / 2 & \text { temps moyen de parcours }
\end{array}
$$

- du profil des vitesses influencé par le nombre d'alignements droits amont et aval et caractérisé par la constante $k$;

- des grandeurs géométriques qu'il est nécessaire de bien connaître ; le débitmètre est fortement sensible aux paramètres géométriques suivants :

- le diamètre intérieur $\mathrm{D}$ de la conduite au droit de la section de mesure :

- la longueur L entre les sondes ultrasonores ;

- la distance d entre les plans contenant les sondes le long d'une génératrice.

Les écarts sur $L, D$ et $d$ se répercutent sur $Q$. Les différents facteurs géométriques influencent le débit d'une quantité proportionnelle à :

$$
\Delta Q / Q=2 \cdot \Delta D / D+2 \cdot \Delta L / L-\Delta d / d .
$$

Le système de mesure à ultrasons délivre deux types de signaux :

- des «tops ", c'est-à-dire des contacts secs, à des intervalles définissant un volume élémentaire transité. Le réglage de cette valeur est accessible à l'utilisateur ;

- une sortie boucle de courant 4-20 mA proportionnelle au débit transitant.

La valeur contractuelle entre les Sociétés est le débit " tops ", calculé à partir des tops et des intervalles de temps entre ces tops, les comparaisons ont été faites sur ce seul débit. Cette sortie impulsionnelle sert aussi à la gestion et à l'exploitation des réseaux. Nous appellerons débit US ce débit calculé à partir des tops.

Enfin, il est à noter une différence significative au niveau du matériel testé. En effet, le paramétrage $(L, D$ et d) de ces appareils se fait :

- pour l'ancien modèle UF 320, à l'aide d'un simulateur sans accès direct aux paramètres;

- pour la dernière version, UF 321, par l'entrée directe de $L, D$ et $d$ sur un clavier intégré.

\section{Les interventions}

\subsection{Déroulement des essais}

Les interventions se sont succédées de mars 1992 à janvier 1993.
Celles concernant les contrôles géométriques étaient locales et rapides.

Pour les essais de comparaison par moulinets, la préparation était plus conséquente. Le montage et les essais duraient une semaine, d'autant plus que se greffaient les problèmes de désinfection; les tuyaux étaient donc « en culture " pendant trois jours. Les essais proprement dits ne prenaient qu'un à deux jours.

L'application de la méthode thermodynamique aux pompes de la station d'Ivry était moins lourde ; montage et essais n'ont duré que trois jours.

Lors de la préparation des essais, il a fallu prendre diverses dispositions concernant la sécurité du personnel dans les conduites (maillage du réseau, liste des vannes à fermer...). Nous avons observé de près les contraintes de salubrité et de désinfection (choix de l'huile de graissage des roulements des moulinets, port de tenues propres, désinfection...).

\subsection{La procédure d'essai}

Sur les 18 ponts qui enjambent la Seine, nous avons réalisé des mesures aux moulinets sur quatre conduites. En diamètre et en volume transité, elles forment un échantillon représentatif de toutes les autres. Leur diamètre varie de 800 à $1500 \mathrm{~mm}$. En ce qui concerne leur implantation, le nombre de longueurs droites en amont et aval est soit conséquent (jusqu'à 150 diamètres), soit réduit à une valeur inférieure aux spécifications données par les notices des débitmètres ultrasonores. La mesure comparative par méthode thermodynamique a permis d'étalonner un débitmètre installé dans une section de diamètre $1100 \mathrm{~mm}$.

L'écoulement s'est toujours fait de Rive Gauche vers Rive Droite, sens le plus souvent utilisé par l'exploitation.

La procédure d'essai avait pour but de mesurer les écarts entre les US et les étalons (moulinets ou thermodynamique) sur la plus large gamme de débit qu'il était possible d'obtenir durant les essais.

Il a fallu bien sûr synchroniser les mesures effectuées par moulinets ou thermodynamique avec les enregistrements des tops issus des débitmètres ultrasonores.

La durée de chaque mesure était de l'ordre de deux à quatre minutes.

Les moulinets étaient placés le plus près possible des ultrasons sans toutefois les perturber. Ainsi, dans l'éventualité d'une fluctuation du débit pendant la durée des essais, les deux sections de mesure intègrent toutes deux cette variation de débit. De plus, grâce aux moulinets, on peut connaître le profil des vitesses au droit de la section ultrasonore (voir encadré (1) et courbes fig. 2).

Avant les essais, la S.A.G.E.P. complétait les mesures en vérifiant le zéro hydraulique (conduite à l'arrêt), la linéarité de la sortie et la célérité du son en eau morte. De plus, la personne responsable réglait le totalisateur de façon à obtenir des tops pour un volume transité de l'ordre du $\mathrm{m}^{3}$ (de 1 à $6,25 \mathrm{~m}^{3}$ selon les cas).

\subsection{Stabilité du débit en essai - Mesures palliatives}

La stabilité des débits lors d'un essai est un problème intrinsèque à un réseau de distribution.

Le réglage d'un débit stable sur un réseau d'eau n'est pas aisé. Selon l'heure, la consommation n'est jamais la 


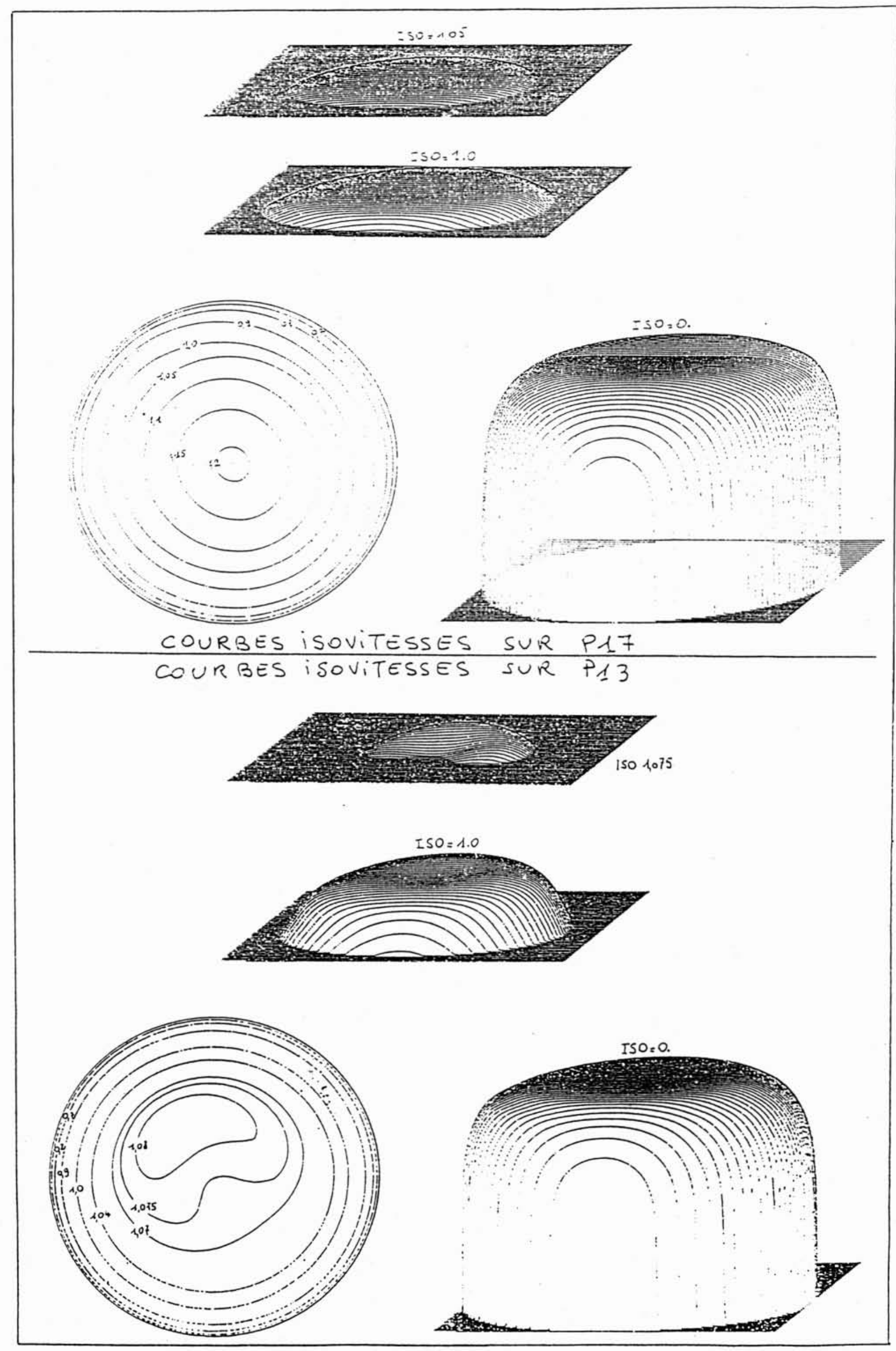

2. Courbes isovitesses sur P17 et P13. 


\section{PROGRAMME MOULINETS - CRITÈRES DE STABILITÉ}

Le programme Moulinets prend en compte toutes les recommandations de la norme NF ISO 3354. Lors d'une campagne d'essais, il permet de s'affranchir de problèmes de mesures en suivant la stabilité du débit. Pendant un essai, le programme réalise des scrutations partielles sur le nombre de tours effectués par les hélices et le temps correspondant. Il effectue ensuite les tests suivants sur ces valeurs intermédiaires pour chaque point de la section de mesure :

soit: $n_{i}$ le nombre de tours effectués par une hélice à la scrutation $i$

$t_{i}$ le temps depuis le début de l'essai correspondant à la scrutation $i$

$N$ le nombre total de scrutations

le programme calcule

$$
\begin{aligned}
& \left.\begin{array}{l}
d n_{i}=\left(n_{i+1}-n_{i}\right) \\
d t_{i}=\left(t_{i+1}-t_{i}\right)
\end{array}\right\} \\
& \omega_{i}=d n_{i} / d t_{i} \\
& \omega=1 / N \cdot \Sigma\left(d n_{i} / d t_{i}\right)
\end{aligned}
$$

Tous ces termes permettent de quantifier la stabilité de l'écoulement, en un point de la section de mesure.

Les termes de vitesse de rotation de l'hélice sont ensuite transformés en vitesse d'écoulement du fluide à l'aide des équations d'étalonnage obtenues dans le canal E.D.F.-D.T.G, de Grenoble,

Des calculs analogues de coefficients de variation sont effectués sur les valeurs de vitesse de l'écoulement et caractérisent la conservation de la forme de l'écoulement lorsque le débit varie. Ces valeurs nous permettent de suivre les moulinets dans le temps. Et, lorsqu'un moulinet a un comportement anormal, on peut corriger sa valeur en appliquant à l'écoulement une loi de conservation de la forme de l'écoulement.

A l'aide des acquisitions réalisées pendant toute la durée des essais, le programme permet en outre de tracer en 2D ou 3D les courbes iso-vitesses (voir fig. 2). Ces courbes permettent de caractériser la forme de l'écoulement et sont très utiles, par exemple pour P13 où les différentes singularités amont et aval rendent le profil légèrement déformé.

même. Nous avons ainsi réalisé les essais à différentes heures de la journée pour obtenir le plus fort débit accessible, c'était généralement tôt le matin.

Ensuite, l'exploitant s'est arrangé pour stabiliser le débit dans notre maille du réseau en réglant judicieusement des vannes alentours.

Nous avons pris des garanties suffisantes pour effectuer les essais dans d'excellentes conditions en s'affranchissant le plus possible de ces problèmes de stabilité. Avant tout, la comparaison s'effectuait sur un temps suffisamment long pour diminuer les incertitudes aléatoires. Mais il doit être assez court car le débit ne peut rester stable longtemps. Il a fallu à chaque fois trouver un compromis.

Ensuite, le moulinet, de part sa faible inertie, réagit aux changements de débit de façon rapide. De même, les US donnent une valeur de volume transité multiple de l'incrément de volume prédéfini, et donc intègrent le débit directement. Enfin, les critères de stabilité calculés par le logiciel de pilotage et de traitement (qui sont explicités dans l'encadré (1)) permettent de signaler des variations de débit trop importantes.

\subsection{Investigations complémentaires}

Compte tenu de la forte influence des paramètres géométriques sur le calcul du débit ultrasonore, nous avons réalisé des essais complémentaires.

Les différences constatées entre les valeurs théoriques de $D, L$ et $d$ et les valeurs réelles mesurées par nos soins sont importantes. Il était donc utile et intéressant d'effectuer quelques essais en rentrant dans les débitmètres US les paramètres géométriques réels de la conduite. Ceci a été fait sur les ponts P13 et P18 et sur la section de mesure D9 en périphérie de Paris.

Dans le même esprit, nous avons cherché à mesurer l'influence de la position des sondes dans l'écoulement. Sur le pont $\mathrm{P} 17$ de petit diamètre et sur $\mathrm{P} 18$ de diamètre le plus grand disponible, nous avons simultanément enfoncé (dans l'écoulement) et retiré (dans le métal) ces sondes par rapport, à la fois à leur position initiale (pas toujours idéale) et par rapport à la position normalisée.

\section{Les étalons de mesure : moulinets et thermo- dynamique}

\subsection{Méthode de jaugeage aux moulinets}

Cette méthode absolue de mesure de débit est précisée en détail dans la norme internationale NF ISO 3354 (réf. biblio [1]). Nous en présentons les points importants dans l'encadré \{2\}. Les essais effectués sont en conformité avec cette norme sauf en ce qui concerne le nombre d'alignements droits pour P13.

Pour les quatre ponts, les mesures de débit ont été réalisées à l'aide d'une section de jaugeage fixe comprenant 13 moulinets équipés d'hélices au pas de $0,25 \mathrm{~m}$ et de diamètre $50 \mathrm{~mm}$, disposés dans la conduite. La structure de la section-type de jaugeage ainsi que la répartition des moulinets sont schématisées sur la figure 3.

La section de jaugeage était implantée dans un alignement droit de la conduite, le plus long possible. Seule la section installée sur le pont $\mathrm{P} 13$ ne respectait pas tout à fait la recommandation du code (alignements droits de 17,8 et 4,7 diamètres respectivement en amont et en aval pour 20 et 5 recommandés).

La section moulinets a été placée à l'aval (a minima à 5 diamètres) de la corde $U S$ pour éviter le plus possible de perturber la mesure $U S$.

Le calcul du débit par moulinets permet en outre de donner le profil des vitesses dans la section de mesure (voir fig. 2). Pour P13, en vue d'obtenir le profil des vitesses, la section moulinets a été placée très proche des $U S$. 


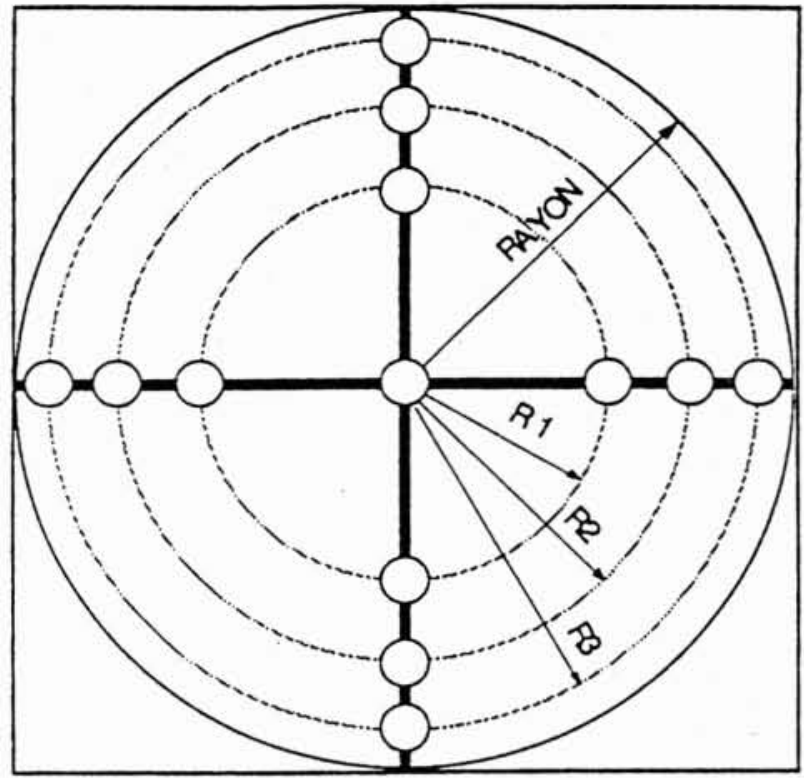

3. Structure de la section-type de jaugeage et de la répartition des moulinets.

Compte tenu de l'interposition de moulinets dans la conduite, pour tenir compte de l'obstruction relative de l'écoulement, nous avons corrigé, conformément à la norme, la valeur brute issue du calcul en la diminuant d'un coefficient correcteur compris entre 0 pour la grosse conduite et $-0,54 \%$ pour les conduites de faible diamètre. On trouvera dans l'encadré $\{2\}$ une formulation de ce coefficient correcteur.

Les essais respectant cette norme donnent une incertitude de mesure maximale de $\pm 2 \%$.

\subsection{Méthode thermodynamique}

La méthode thermodynamique applique le principe de la conservation de l'énergie (première loi de la thermodynamique) à l'échange d'énergie entre l'eau et la machine qu'elle traverse.

On accède directement au débit en mesurant des grandeurs thermodynamiques (pression, température, vitesse, niveau) et en utilisant les propriétés thermodynamiques de l'eau (voir encadré $\{3\}$ ci-contre).

En mesurant accessoirement la hauteur nette de refoulement de la pompe, on accède au rendement de la machine. Nous avons donc en plus de la détermination du débit, tracé la courbe de rendement de la pompe P5. Elle se trouve sur la figure 4.

Notre référence est la norme CEI 41 (réf. biblio (2)).

Les essais effectués sur la pompe P5 de la station d'Ivry respectent cette norme sauf pour la hauteur nette de refoulement qui doit rester supérieure à $100 \mathrm{~m}$.

Cependant, compte tenu des excellentes conditions des essais, et des garanties que nous avons prises, on peut considérer que l'incertitude de mesure sur les débits reste dans la fourchette donnée par la norme, et est inférieure à $1,5 \%$.

\section{Encadré 2 \\ MESURES DE DÉBIT PAR MOULINETS - NORME NF ISO 3354}

La norme NF ISO 3354 de décembre 1988 définit la « méthode d'exploration du champ des vitesses dans les conduites en charge et dans le cas d'un écoulement régulier, au moyen de moulinets ».

Les moulinets sont constitués d'un corps fixé sur une structure et d'une hélice se « vissant "dans l'écoulement. Ce sont des hélices à l'emballement servant à mesurer une vitesse locale d'écoulement d'un fluide. Le moulinet est défini par son diamètre (50 à $125 \mathrm{~mm}$ ) déterminant son utilisation en petite ou grande conduite, et son pas $(0,25$ et $0,50 \mathrm{~m})$, qui est le déplacement du fluide occasionnant un tour entier de l'hélice.

Ce pas reste théorique, et compte tenu de l'imperfection des matériels, il faut réaliser un étalonnage. Cette opération est effectuée en nos locaux dans un canal approprié de $100 \mathrm{~m}$ de long. Elle donne la correspondance entre la vitesse de rotation du moulinet et la vitesse du fluide au voisinage de celui-ci.

Le débit est déterminé par intégration numérique du champ des vitesses. L'interpolation entre les points de mesure se fait suivant des courbes du troisième degré. Dans la zone comprise entre la paroi et les moulinets les plus excentrés (appelée zone pariétale), on admet une loi polynomiale de répartition des vitesses en fonction de la distance à la paroi, de puissance $m$, facteur appelé coefficient de couche limite.

La présence dans la conduite de moulinets et de leur support entraîne une réduction de la section de passage de l'écoulement et donc une altération de la répartition des vitesses. Le phénomène est entièrement différent lors de l'étalonnage des moulinets dans le canal où existe une surface libre et où les moulinets à étalonner sont suffisamment éloignés des parois. Le calcul du débit dans une conduite sur la base de l'étalonnage déterminé en canal, conduit donc à surestimer le débit.

On introduit alors une correction $k$ diminuant le débit d'une valeur fonction des encombrements relatifs des perches support $s$ et des moulinets $s_{c}$ dans la section. Ces encombrements relatifs se calculent par le rapport du maître couple des organes dans l'écoulement à l'aire de la section de mesure. On obtient $k$ en \% par :

$$
\begin{aligned}
& k=0,12 \cdot s+0,03 \cdot s_{c} \text { si } s>2 \% \\
& \text { sinon } k=0
\end{aligned}
$$

Pour les sections de mesure moulinets en conformité avec cette norme, le débit sera connu avec une incertitude de mesure au plus égale à $\pm 2 \%$, à condition que la correction correspondant à l'effet d'obstruction soit apportée.

\section{Encadré 3}

\section{CALCULS THERMODYNAMIQUES}

En raison des difficultés à les mesurer directement dans l'écoulement, les grandeurs définissant l'énergie mécanique massique $E_{m}$ sont mesurées dans des enceintes spécialement aménagées pour la mesure de température $T$ et pression $P$.

On réalise un soutirage de débit $(\approx 0,5 \mathrm{l} / \mathrm{s})$ par une prise dynamique.

L'énergie $E_{m}$ se calcule par :

$$
E_{m}=a \cdot \Delta P+C_{p} \cdot \Delta T+g \cdot \Delta Z
$$

avec $a$ et $C_{p}$ coefficients thermodynamiques

et $g$ accélération de la pesanteur

En mesurant en plus la hauteur nette de refoulement $H$ :

$$
H=E / g=\Delta P^{\prime} /(\rho g)+\Delta\left(V^{2} / 2\right) / g+\Delta Z^{\prime}
$$

on accède au rendement de la pompe :

$$
\eta=E / E_{m}
$$

si on néglige les pertes mécaniques. 


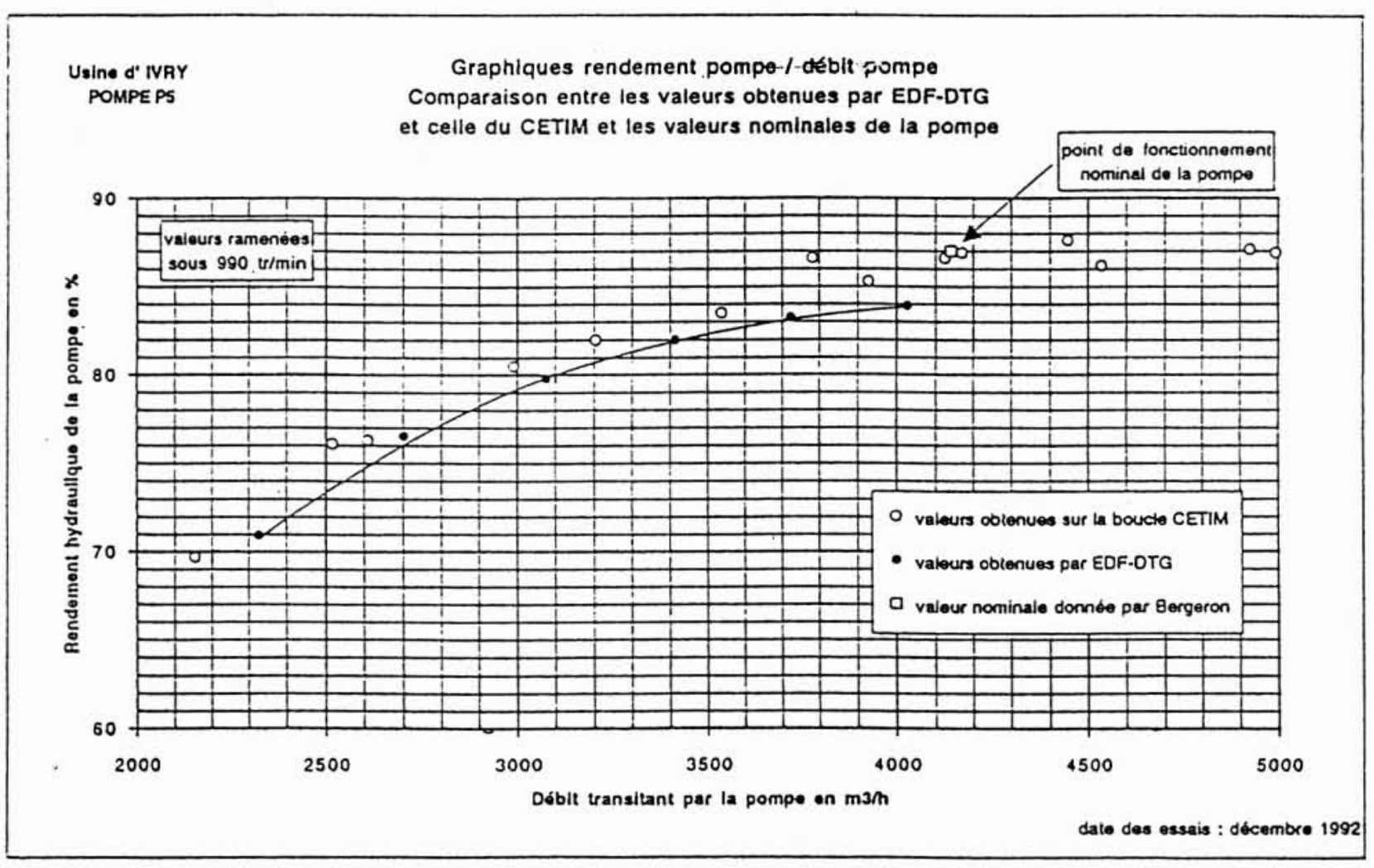

4. Courbe de rendement pompe/débit pompe de l'usine d'Ivry.

\section{Les résultats}

\subsection{La géométrie}

Les recommandations d'installation d'Ultraflux stipulent qu'il est nécessaire de bien connaître la géométrie de la section de mesure. L'expert souhaitait donc voir ce qu'il en était.

Les mesures ont été réalisées au total sur 30 sections de mesures entre les ponts enjambant la Seine et les sections implantées en périphérie. Toutes les sections étaient équipées de débitmètres de la marque Ultraflux UF 320 ou UF 321 ; elles étaient toutes instrumentées de monocordes, sauf un bicorde sur la section D13.

Cinq paramètres ont été mesurés par des moyens appropriés (réglets, vis micrométriques, constructions géométriques) :

- le diamètre intérieur $D$ moyen de la conduite au centre des sections contenant les sondes;

- la distance physique $L$ entre les faces de sondes ultrasonores ;

- la distance $d$ sur une génératrice entre les sections contenant les sondes ;

- l'enfoncement des sondes par rapport à la position normalisée ;

- enfin nous avons vérifié que la corde passait bien par l'axe de la conduite.

Toutes ces mesures sont reprises sur la figure 5 ci-après. Les résultats montrent des écarts conséquents allant jusqu'à $3 \%$ sur $D, 3,9 \%$ sur $L$ et $4,1 \%$ sur $d$. Quelques sondes ont été trouvées bien positionnées. La plupart étaient décalées de quelques $\mathrm{mm}$. Nous avons mesuré des écarts allant jusqu'à $68 \mathrm{~mm}$ de retrait dans le métal et des sondes saillant de $29 \mathrm{~mm}$ dans l'écoulement. La figure 6 montre un exemple flagrant de ce que nous avons observé : dans une section revêtue en surface, les sondes sont bien installées par rapport à la conduite en métal mais l'épaisseur du revêtement fait qu'elles se retrouvent en retrait de la position normalisée.

Rappelons ici la formule (1) du calcul du débit ultrasonore :

$$
Q=k \cdot\left(\Delta t / t^{2}\right) \cdot L^{2} \cdot D^{2} / d .
$$

Les variations de $L, D$ et $d$ se répercutent sur $Q$ en

$$
\Delta Q / Q=2 \cdot \Delta D / D+2 \cdot \Delta L / L-\Delta d / d .
$$

Ainsi, un écart de $1 \%$ sur le diamètre donne une erreur sur le débit de

$$
\Delta Q / Q=2 \cdot \Delta D / D=2 \% .
$$

Contrairement à ce qui est précisé sur la notice de montage Ultraflux, ces débitmètres ont été installés sans recours à une vidange sur une conduite dont on ne connaissait pas les dimensions exactes. Les sondes ont été mises en place depuis l'extérieur, sans mesure ou observation interne. Les paramètres entrés $(D, L$ et $d)$ dans les débitmètres sont donc les valeurs théoriques des cotes des conduites. Aucune vérification n'a été réalisée sur la qualité d'installation des sondes ultrasonores. 


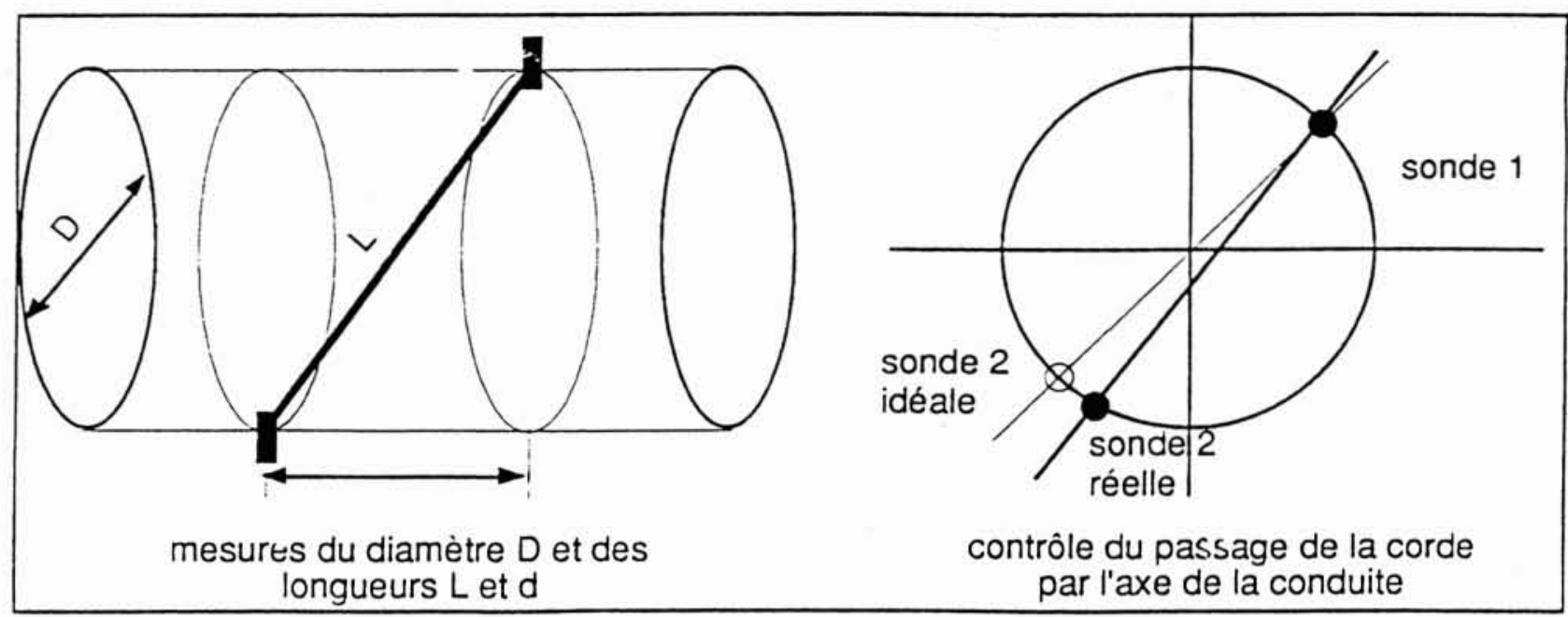

5. Mesure des paramètres géométriques de la section de mesure.

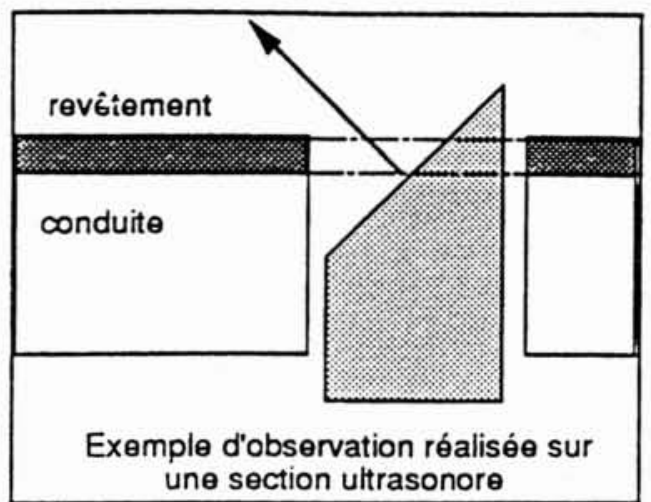

6. Exemple d'observation réalisée sur une section ultrasonore.

\subsection{Les divers essais comparatifs}

Les résultats bruts sont donnés sur les courbes de la figure 7. Ils sont présentés sous forme de courbes donnant l'allure des variations des écarts en fonction du débit de référence, ceci pour les ponts $\mathrm{P} 2, \mathrm{P} 13, \mathrm{P} 17$ et P18. Nous avons rajouté les essais comparatifs par la méthode thermodynamique qui ont permis d'étalonner le débitmètre $U S$ installé sur la section D9.

La différence débit tops US/référence s'entend :

$$
\begin{gathered}
\Delta=[(\text { débit tops } U S)-(\text { débit référence })] /(\text { débit référence }) \\
\text { en } \% .
\end{gathered}
$$

Les premières conclusions montrent une grande disparité des mesures, les écarts allant du sur-comptage systématique de $+5,5 \%$ à un sous-comptage important proche de $-18 \%$.

Aucun des débitmètres installés ne donne un débit correct sur toute la gamme des débits mesurés :

- le débitmètre installé à Pont de Conflans P2 surestime le débit mesuré par les moulinets. L'écart entre les deux mesures varie de $+1 \%$ à $+2 \%$ pour des débits faibles (de 0,06 à $0,1 \mathrm{~m}^{3} / \mathrm{s}$ ), jusqu'à atteindre $+5 \%$ pour des débits compris entre 0,15 et $0,4 \mathrm{~m}^{3} / \mathrm{s}$;
- le débitmètre P13 du Pont Royal sous-estime le débit mesuré par les moulinets, avec un écart entre les deux mesures proche de $-18 \%$ à $0,15 \mathrm{~m}^{3} / \mathrm{s}$ et égal à $-13 \%$ de 0,3 à $0,7 \mathrm{~m}^{3} / \mathrm{s}$;

- le débitmètre US du Pont du Garigliano P17 surestime le débit de référence sur une large gamme de débit jusqu'à un écart de $+5,5 \%$. A faible débit, le débitmètre $U S$ sous-estime le débit de référence jusqu'à $-0,5 \%$;

- sur le même pont, le débitmètre P18 surestime le débit sur une large gamme. La valeur de l'écart entre les moulinets et les ultrasons varie de $-1,5 \%$ à faible débit (souscomptage) jusqu'à $+4 \%$ pour des débits de l'ordre de 0,6 et $1,2 \mathrm{~m}^{3} / \mathrm{s}$ (sur-comptage) ;

- pour D9, le débitmètre sous-compte sur toute la gamme des débits mesurés entre $-0,55 \%$ et $-1,83 \%$ si les valeurs théoriques de la géométrie sont entrées en configuration.

Lors de l'étalonnage de la section D9, nous avons réalisé la courbe de rendement de la pompe P5 de l'usine de pompage d'Ivry. Nos résultats donnent une courbe de rendement qui figure en (8). Nos mesures n'ont pu être facilement comparées aux essais précédents.

Concernant la comparaison tops US/4-20 mA, les différences s'étagent de $-4,5 \%$ à $+4,5 \%$ pour l'ensemble des ponts, les écarts les plus significatifs étant réalisés à bas débit.

Ces résultats bruts doivent être analysés de plus près. Nous avons donc réalisé divers essais complémentaires. Ils expliquent en partie les écarts importants mesurés.

\section{Les écarts constatés - Quelques explications}

\subsection{Géométrie réelle}

Les écarts constatés sont très importants, largement supérieurs à l'incertitude de mesure des étalons. On note pourtant que les conditions d'alignements droits sont presque parfaites sauf pour P13 où on observe les plus grands écarts. Les causes ont été cherchées ailleurs. Bien entendu, les seules causes analysées concernent la mise en œuvre des ultrasons, considérant que nous avons appliqué les étalons selon la norme. 


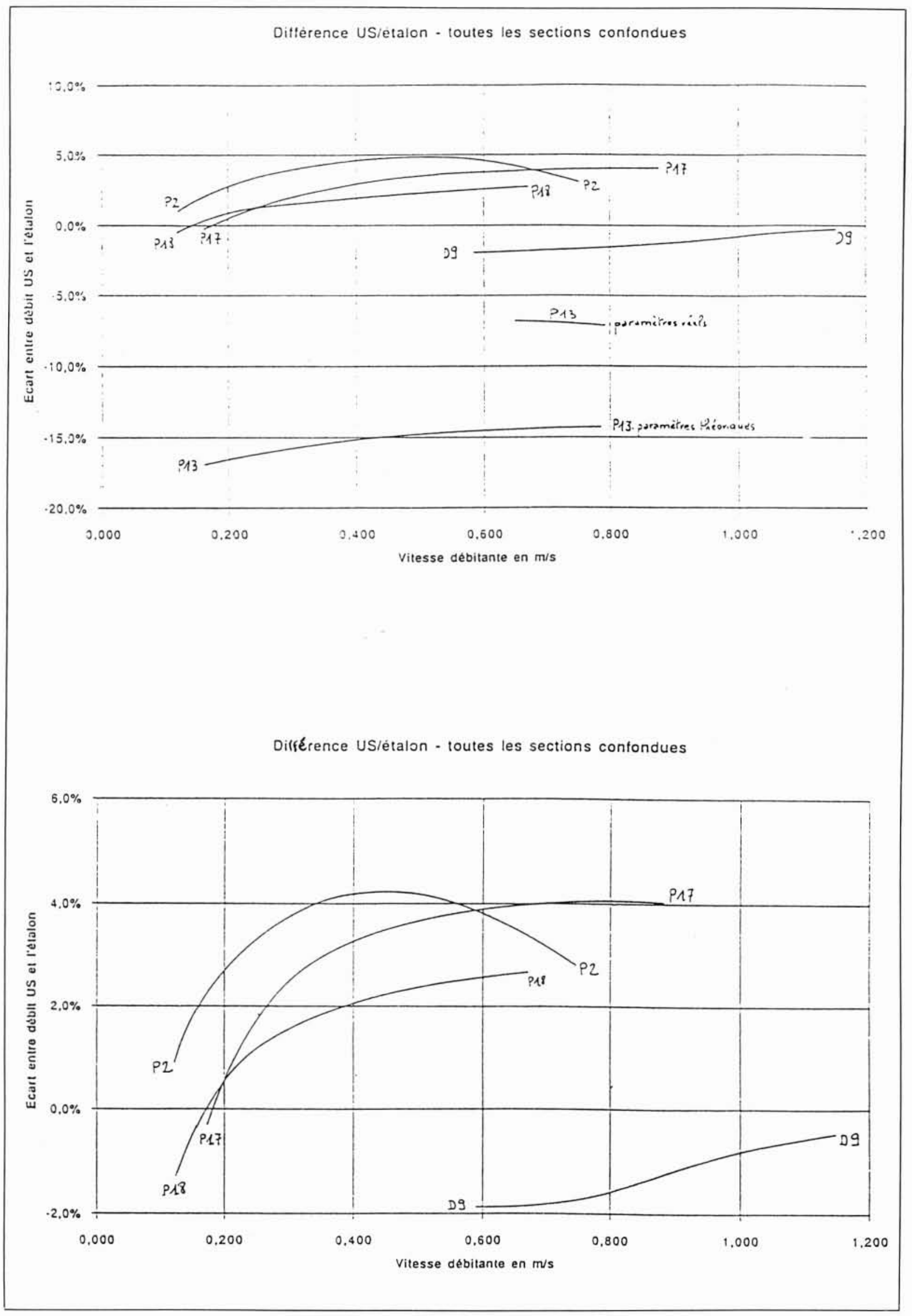

7. Écart entre débit $U S$ et étalon. 
Nous avons alors réalisé sur trois ponts des mesures comparatives en paramétrant les débitmètres successivement avec les paramètres géométriques théoriques puis les paramètres réellement mesurés.

- Sur P18, deux essais ont été effectués en introduisant dans le débitmètre les paramètres mesurés. Ces essais n'ont pas donné de modification significative de l'écart US/moulinets. Le débitmètre placé sur ce pont est de modèle UF 320. Il faut donc entrer les coefficients à l'aide d'un simulateur et il n'est pas possible d'afficher les paramètres introduits.

L'écart attendu n'est pourtant pas négligeable puisqu'il atteint $-2,3$ points.

- Sur P13, le débitmètre est de type UF 321. Les paramètres mesurés ont donc pu être entrés et vérifiés. Lors des deux essais effectués, la différence avec/sans modification varie considérablement pour atteindre +7 à +8 points. Cette différence correspond à la valeur qu'on est en droit d'attendre de cette modification, c'est-à-dire de $+8,0$ points.

- Sur D9, on remarque que, pour le débitmètre ultrason, l'écart entre les mesures avec la géométrie théorique puis la géométrie réelle est pratiquement constant et très voisin de $-1,7$ point ; cette valeur est celle apportée a priori par l'entrée des nouveaux paramètres, soit $-1,7$ point aussi.

Ainsi, dans les cas où les paramètres sont accessibles directement (UF 321), les écarts constatés entre les mesures avant et après modification des paramètres géométriques sont en complète adéquation avec les calculs théoriques.

\subsection{Enfoncement des sondes}

La notice d'utilisation des débitmètres Ultraflux spécifie une position normalisée des sondes dans l'écoulement.
Leur centre doit affleurer la paroi de la conduite.

Une fois de plus, suite aux mesures géométriques que nous avons réalisées et où nous avions trouvé des positions de sondes inadéquates, nous avons cherché à évaluer l'influence de cette position des sondes dans la conduite.

Le changement physique de la position des sondes affectera les paramètres du débitmètre. Ces changements touchent en premier lieu la distance entre ces sondes, paramètre que nous n'avons pas modifié dans l'appareil, mais en plus l'angle de visée des sondes ultrasonores. Enfin, si on enfonce les sondes dans l'écoulement, on ne prend plus en compte l'intégralité du profil des vitesses sur tout le diamètre, et une zone pariétale est oubliée dans le calcul. On observe un changement non négligeable des sorties US et les écarts varient en fonction de la position des sondes:

- si on enfonce les sondes dans l'écoulement, le débitmètre calcule un débit plus fort que la position normale ;

- si on les retire dans le métal, le débit sera sous-estimé mais d'une façon plus sensible que le même déplacement dans l'écoulement.

Pour vérifier cette théorie élaborée par M. ANDRÉ, des essais ont été réalisés sur deux conduites d'un même pont, $\mathrm{P} 17$ et P18.

Sur P17, les résultats font ressortir la tendance suivante : - lorsque les sondes sont dans leur état initial, l'écart tops US/moulinets est de l'ordre de $+3 \%$;

- lorsque les sondes sont enfoncées (dans l'écoulement), l'écart augmente jusqu'à 5\%;

- quand les sondes sont retirées (dans le métal), l'écart diminue jusqu'à $0 \%$.

Les mesures réalisées sur P17 montrent la tendance donnée par la théorie ; malheureusement, voir figure 8 , on ne peut faire sortir de loi de comportement, les résultats étant trop irréguliers.

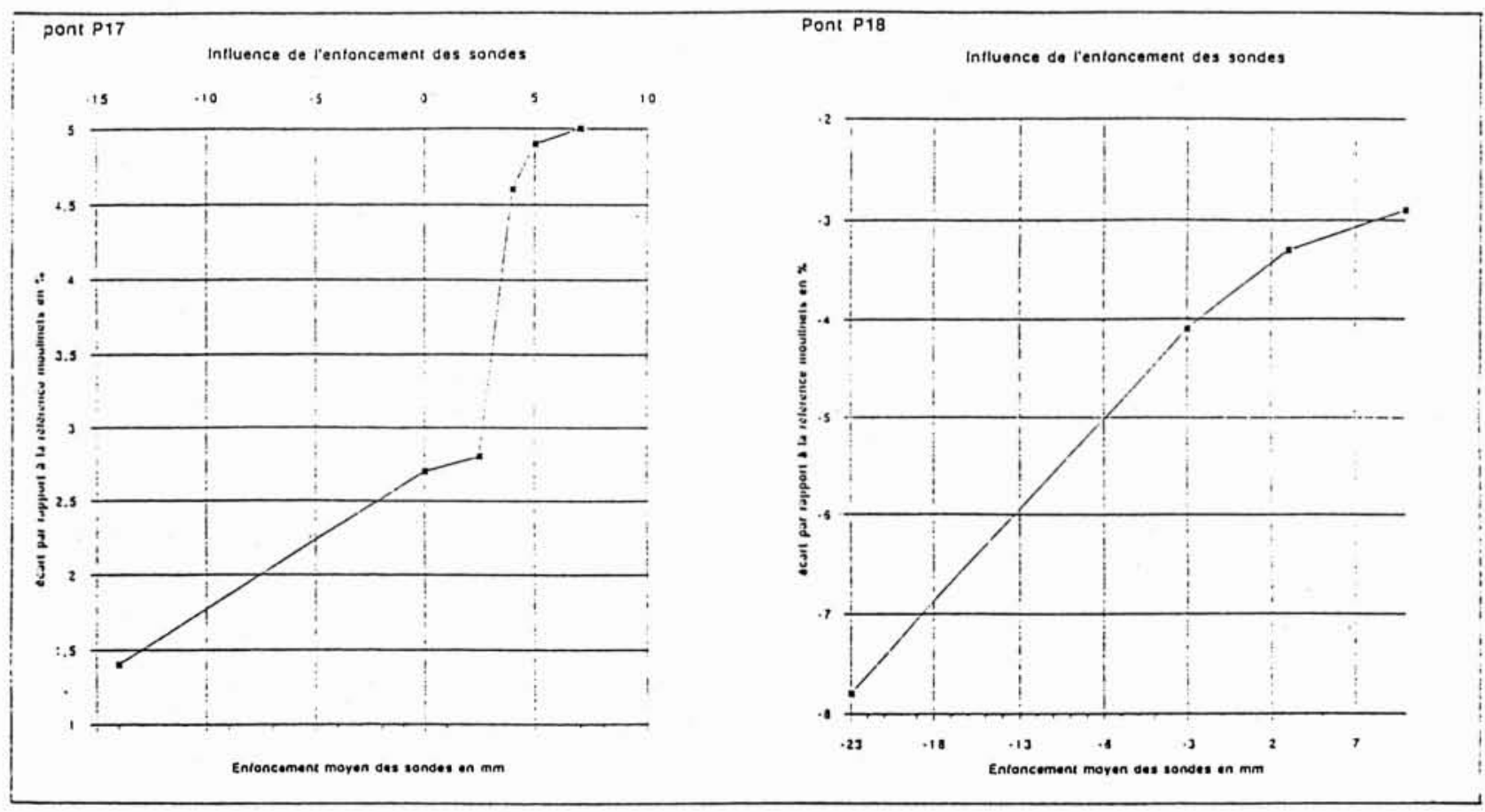

8. Influence de l'enfoncement des sondes. 
Nous avons alors réalisé une autre campagne d'essais sur la conduite P18 instrumentée lors de cette campagne d'un UF 321 de nouvelle génération plus précis que le modèle analogique UF 320 installé sur P17 et anciennement sur cette section P18.

Quatre séquences d'essais ont été réalisées successivement pour trois débits différents, avec des positionnements de sondes US différents.

Les essais montrent l'influence de la position de ces sondes sur le calcul du débit par ultrasons. On trace en figure 8 les évolutions des valeurs moyennes des écarts par rapport à la position normalisée. Dans les cas de P18, on retrouve non seulement l'évolution théorique, mais on atteint les écarts attendus par la théorie.

En effet, lorsque les sondes sont en retrait dans le métal, le débitmètre voit toujours le même écoulement au total, donc mesure toujours le même $\Delta t$ (voir (1)) entre les deux temps de parcours. La longueur $L$ entre les sondes change. Mais, lors des essais, nous n'avons pas corrigé cette valeur dans le paramétrage du débitmètre. Ce changement de longueur entre les sondes influence le terme $t$ de temps de parcours ; dans le cas de $t$, cette grandeur mesurée par le débitmètre change la valeur du débit. L'angle de tir est lui aussi modifié même s'il reste très proche de $45^{\circ}$. La correction totale prend en compte tous ces termes.

Sur P17, les résultats ne suivent pas cette théorie. Par contre, sur P18, on obtient :

- pour un retrait de $3 \mathrm{~mm}$, un écart mesuré de $-0,4 \%$ pour $-0,6 \%$ en théorie :

- pour un retrait de $23 \mathrm{~mm}$, un écart mesuré de $-4,1 \%$ pour $-4,6 \%$ en théorie.

Lorsque les sondes sont saillantes dans l'écoulement, elles ne prennent pas en compte les faibles vitesses proches de la paroi. Le débitmètre mesure par contre un temps $t$ moyen plus faible car les sondes sont plus rapprochées. Le calculateur mesure donc une vitesse plus forte et l'applique à toute la section mouillée. Il y a surestimation du débit.

On observe que l'influence de l'enfoncement des sondes dans l'écoulement est moindre en valeur absolue qu'un même déplacement en retrait dans le métal.

En utilisant les profils de vitesse déterminés par le programme moulinets, on peut retrouver la vitesse moyenne de l'eau calculée par le système à ultrasons entre les deux sondes, et donc le débit calculé par la baie de mesure US.

En adoptant pour la partie de l'écoulement proche de la paroi un profil de vitesse en loi polynomiale, et en calculant l'erreur due au changement dans l'angle de visée, on obtient un écart théorique très proche de celui mesuré ; par exemple pour P18, un enfoncement de $3 \mathrm{~mm}$ donne une valeur mesurée de $+0,4 \%$ pour $+0,5 \%$ en théorie ; enfin, pour un enfoncement de $10 \mathrm{~mm}$, on obtient un écart mesuré de $+0,8 \%$ pour $+1,4 \%$ calculé.

Les résultats sont moins précis pour l'enfoncement des sondes dans l'écoulement, mais les écarts relevés sont du même ordre de grandeur que la théorie.

\subsection{Réponses complémentaires}

Les corrections de géométrie et d'enfoncement des sondes ne sont donc pas suffisantes pour expliquer la totalité des écarts constatés.

L'écart reste important pour P13, où les sondes sont bien positionnées, et où l'écart reste de 6 à $7 \%$ avec les moulinets lorsque les coefficients réels de géométrie sont entrés. Nous avons effectué sur cette section de mesure un calcul complémentaire. Ce calcul montre que, en ne considérant que les moulinets implantés sur la diagonale de la corde $U S$, le débit vu par la corde US est pratiquement égal à celui calculé avec les informations des 13 moulinets. Ainsi, sur trois essais répartis sur la gamme des débits mesurés, l'écart est presque nul $( \pm 0,7 \%)$.

L'observation du profil des vitesses entre P13 et le pont P17 installé sur de bons alignements droits montre des différences significatives, mais pas suffisantes pour montrer que la corde US est mal implantée.

\subsection{Expertise de l'électronique - Erreur imputable au maté- riel}

La S.A.G.E.P. a contacté E.D.F.-D.T.G. pour réaliser l'expertise de l'appareil du point de vue de l'électronique. Cette intervention a été réalisée dans nos laboratoires sur l'appareil dernier cri d'Ultraflux : I'UF 321.

Le cœur de l'appareil est un compteur destiné à mesurer le temps de vol de l'onde ultrasonore entre la céramique d'émission et celle de réception. La résolution désirée est de $1 \mathrm{~ns}$ (nanoseconde), ce qui est beaucoup demander à une électronique réalisée en composants classiques (fig. 9).

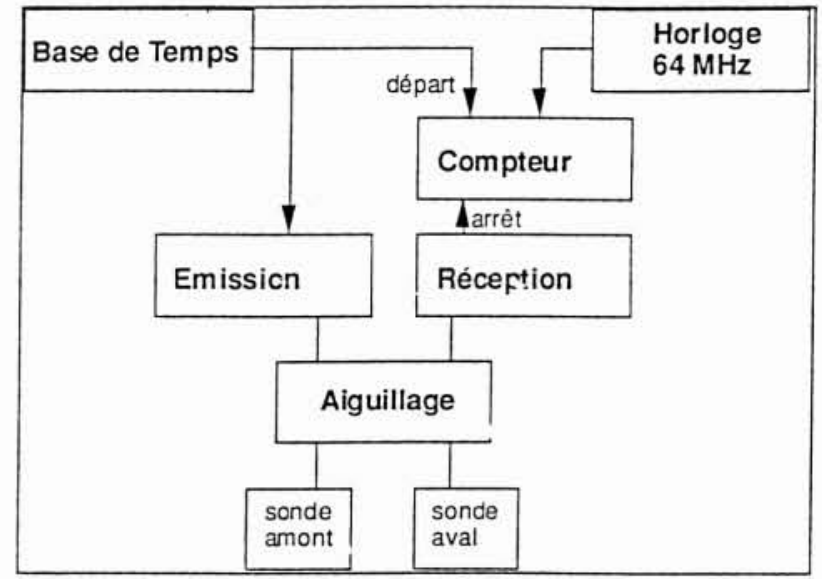

\section{Electronique de l'appareil UF 321.}

Un amplificateur à gain variable permet d'obtenir un signal d'amplitude suffisante et d'éviter la saturation indépendamment de la section de la conduite d'eau, donc du trajet parcouru par l'onde US.

Le compteur utilise une horloge de période $16 \mathrm{~ns}$. Le déclenchement de la mesure étant aléatoire vis-à-vis de cette horloge, on peut estimer que l'erreur aléatoire est réduite dans le rapport $\sqrt{N}$ après $N$ mesures. Dans cette hypothèse, l'erreur de mesure n'est plus que de 1 ns après 256 mesures.

Différents essais ont été réalisés, soit avec un simulateur contrôlé par des compteurs fréquencemètres de haute performance, soit plus simplement en utilisant de l'eau immobile, ce qui correspond donc à une différence de temps de vol nulle.

Les résultats mettent en évidence une dispersion de mesure de temps pouvant atteindre $20 \mathrm{~ns}$ dans le cas d'un temps de vol voisin de $200 \mu$ s. 
Par ailleurs, les sondes ne sont pas identiques et introduisent une différence d'environ $10 \mathrm{~ns}$ selon le sens de la mesure.

Des essais plus poussés conduisent à considérer que l'appareil a une précision de mesure de l'ordre de $1 \%$ sur les débits cumulés, indépendamment du positionnement des sondes dans la conduite.

Les tests aux perturbations électromagnétiques montrent une bonne résistance aux ondes radio. Les essais, non normalisés, ont été effectués avec des émetteurs portatifs de $1 \mathrm{~W}$ à $150 \mathrm{MHz}$ et $4 \mathrm{~W}$ à $27 \mathrm{MHz}$ placés à $1 \mathrm{~m}$ de l'électronique.

Le débitmètre est par contre très sensible aux décharges électrostatiques (test normalisé) et a cessé de fonctionner correctement après cet essai.

\section{Conclusion générale}

L'expertise des débitmètres $U S$ installés in situ montre des écarts conséquents avec des références étalon mises en œuvre par E.D.F.-D.T.G.

Des explications trouvent leur source dans le paramétrage des baies de mesure, en y entrant les valeurs réelles de la géométrie mesurées auparavant par nos soins. Les mesures réalisées ont en effet montré de grandes disparités dans la pose de capteurs ultrasonores entre les valeurs réelles mesurées et les paramètres entrés dans les débitmètres.

Les erreurs sur les paramètres géométriques et sur le positionnement des sondes dans l'écoulement, en retrait dans le métal ou saillant dans le fluide, influencent grandement les mesures.

Il est donc capital, lors de l'installation de tels systèmes de mesure de prendre toutes précautions utiles et de suivre à la lettre les recommandations d'installation des capteurs. Avant tout, il faut choisir une section de mesure avec de grandes longueurs droites; ensuite mesurer tous les para- mètres entrés dans la baie de mesure et éviter à tout prix d'installer ce système sans vidange et sans accès à l'intérieur de la conduite.

Par contre, en prenant toutes ces précautions et en corrigeant les résultats des erreurs géométriques ou de positionnement, il reste un écart résiduel non négligeable.

L'écart observé sur P13 n'est pas expliqué seulement par la géométrie de l'installation. Même si le débitmètre est implanté sur une faible section droite, une seule corde n'est pas suffisante pour définir complètement et correctement cet écoulement perturbé.

Les écarts résiduels sur les autres ponts bien installés en ce qui concerne le nombre d'alignements droits, doivent trouver une explication ailleurs, par exemple dans le calcul du coefficient hydraulique $k$ du calcul du débit, influencé par le coefficient de pertes de charge, donc par la rugosité de la conduite.

De plus, l'expertise de l'électronique du débitmètre UF 321 montre qu'on ne peut s'attendre à une précision meilleure que $1 \%$ sur ce matériel.

Un étalonnage sur place est fortement conseillé.

L'expertise a finalement permis aux exploitants de remettre correctement tout le système de mesure en ordre. Les paramètres géométriques réels ont été introduits dans les débitmètres. Les sondes sont dans leur position normalisée. Les erreurs résiduelles sont bien connues.

\section{Références bibliographiques}

[1] Norme NF ISO 3354 - décembre 1988 - * Méthode d'exploration du champ des vitesses dans les conduites en charge et dans le cas d'un écoulement régulier, au moyen de moulinets $»$.

[2] Norme CEI 41 - novembre 1991 - « Essais de réception sur place des turbines hydrauliques, pompes d'accumulation et pompes-turbines, en vue de la détermination de leurs performances hydrauliques ». 